\title{
Delle Forme di Espressione del Diritto.
}

\author{
R. Limongi França \\ Professore Della Facoltà di Diritto Della Università \\ di San Paolo.
}

\begin{abstract}
Sommário: I) Importanza Dello Studio Delle Costddetre "FonTi" Dei Diritto. II) INFormazione SUlla QUESTione Delle "Fonti Dex Diritro": 1. Scuola Storica. 2. Studi contemporanei particolarmente importanti sulla materia. 3. L'opera di Giny. 4. Contributo dei publicisti. 5. Brethe de la Gressaye e Laborde - Lacoste; III) SosTITUZIONE DELLA IDEA DI "FONTE" CON QUELLA DI "FORMA" DEL DIRITto Positivo: 1. Improprietà della espressione "Fonte" per designare $i$ modi di Espressione del Diritto. 2. L'idea di "fonte formale". 3. Ahrens e Fernandes Elias. 4. Necessità della distinzione tra "fonte" $e$ "for$m a$ " del Diritto. IV) Classificazione Delue Forme DI ESPRESSIONE DEL DIRITTO: 1. Introduzione. 2. Fonti Storiche. 3. Fonti Genetiche. 4. Fonti Strumentali. 5. Forme di Espressione del Diritto. Classificazione secondo il criterio della "natura della coercitività". 6. Classificazione secondo il criterio della "importanza strutturale".
\end{abstract}

\section{Importanza dello studio delle cosiddette "fonti" del Diritto.}

Fonte è il luogo da cui proviene qualche cosa. Fonte del Diritto sarebbe, per analogia, il luogo da cui sono oriundi i precetti giuridici.

Sebbene la precisione e l'origine di questa idea siano discutibili, riteniamo sufficiente presentarla in detti termini, affinchè si possa valutare l'importanza della materia, tanto per quanto concerne l'investigazione pura e semplice dei fatti giuridici, tanto per ciò che riguarda l'applicazione pratica della norma giuridica ai problemi ad essa attinenti, e che fluiscono inevitabilmente dalla complessità della vita sociale. 
In verità, non vi serà professione $o$ attività nella vita del Diritto che non dipenda, per il suo sviluppo, da nozioni basiche collegate al presente argomento, nè vi sarà questione $\mathrm{di}$ natura giuridica che non debba ricercare e trovare nelle "fonti" la soluzione ad essa necessaria.

Le ragioni di um avvocato, il parere di un giureconsulto, il libello di un pubblico ministero, la sentenza di un giudice, linsegnamento di un cattedratico, l'investigazione di uno scienziato del Diritto non potranno mai prescindere dal ricorso diuturno, costante ed impostergabile agli elementi forniti dalle fonti delle relazioni giuridiche.

Malgrado ciò, la bibliografia nazionale, a questo riguardo, è semplicemente poverissima. Evidentemente, la grande maggioranza dei nostri trattatisti si occupa continuamente di detto argomento, che arriva persino ad essere un luogo comune sia nelle opere di teoria generale del Dirito Civile, che in quelle di introduzione alla Scienza del Diritto. Tuttavia, "data venia" non si è notato nessuno sforzo, da parte dei rispettivi autori, per rivedere le dottrine e i concetti sulla materia, sebbene da molto tempo, come si vedrà più innanzi, se ne venga sentendo il bisogno.

Mentre ciò si verifica tra di noi, in altri paesi l'argomento è stato oggetto di meditate ponderazioni, manifestate in monografie di profondo valore filosofico e giuridico, i cui risultati sono di grande importanza per la revisione del tema, che in questo modo, ha la possibilità di compiere sempre più un passo avanti nelle conquiste della Scienza Giuridica.

Lo scopo del presente lavoro, ovviamente, non è quello di "colmare lacune" Tuttavia speriamo, dato il nostro ardente desiderio di rimanere fedeli allo spirito universitario, che esso possa per lo meno delinearne i primi elementi, i rudimenti, affinchè altri (forse, in un tempo futuro, i nostri propri alunni), possano realizzare ciò che, accanto a molte altre 
cose, sta mancando tra di noi: nel caso specifico, uno studio sei io e perfezionato rispetto a quell'importantissimo capitolo che è la propedeutica del Diritto Civile e della Scienza Giuridica in generale.

\section{Informazioni sul problema delle fonti del Diritto.}

1. La Dottrina delle Fonti, Secundo la Scuola Storica. Savigny e Puchta. Fu senza dubbio con la Scuola Storica del Diritto, fiorita agli albori del secolo XIX ${ }^{1}$ e i cui pro-uomini furono Savigny e Puchta, che ebbero inizio i primi studi realmente profondi sulle considdette "fonti" del Diritto.

Effettivamente, prima della Scuola Storica, e specialmente prima di Savigny, fin dal tempo dei Romani, lo studio delle fonti si era limitato alla descrizione esterna, senza alcuna preocupazione di ricercare le origini e le ragioni dell'essere ${ }^{2}$.

1. La Scuola Storica, secondo quanto dimostrò il preclaro maestro ALEXANDre Correa (A Concepção Histórica do Direito, pp. 10-22 e pp. 23-52, São Paulo, 1934) ebbe come suoi alti rappresentanti Burke, in Inghilterra, e De Maistre, in Francia. Tuttavia, fu in Germania che trovò i suoi grandi realizzatori, tra 1 quali spicca la figura gigantesca di SAvignY, la cui opera fu secondata dal suo discepolo Puchta.

Una cosi importante corrente del pensiero giuridico non è altro che un aspectto particolare di tutta una concezione generale, riguardante la nascita e l'evoluzione delle istituzioni umane. Costituisce, dentro del panorama delle Idee filosofiche - giuridiche, una reazione contro la Scuola del "Naturrecht" - del diritto naturale assoluto o giusnaturalismo, che imperò soprattutto nel secolo XVIII.

La sua centrale, secondo quanto si osserva nel Sistema del Diritto Romano Attuale, di Savigny (v. trad. di Scialoja, 1886) e nel Corso delle Istituzioni di Puchta (trad. di Turchiarulo, Napoll, 1854) consiste nella negazione della possibilita di dedurre il Diritto "more geometrico", vale a dire, secondo un processo esclusivo di raziocinio, indipendentemente dalla realtà del fatti. Prima dl lui, è solamente nella evoluzione storica della realtà delle instituzionl giuridiche, cosi come avviene negli idioml, che troviamo l'origine di tutto Il diritto. Esistendo allo stato latente nella coscienza del "popolo" o nella "coscienza nazionale", ha come prima manifestazione il "costume", il cui significato è quello di uno stadio anteriore alla legge.

Come la lingua, il diritto è differente per ogni popolo, e non é più diritto quando non esprime la "opinio necessitatis" della coscienza popolare ( $\$ \S \mathrm{X}$ a XXI, pp. 7-15, del Corso di Рбснта)

2. Saviany, op. cit., p. 115 e segg. 
E, per esempio, quanto si nota nell'opera di Heinecius, della cosiddetta Scuola dei Post-Glossatori ${ }^{3}$, e in quella dei giureconsulti portoghesi del secolo XVIII, tra i quali risalta la considerevole figura di Mello Freire ${ }^{4}$. La stessa opera di Montesquieu, il celeberrimo trattato De L'Esprit des Lois, che gli valse la posizione di precursore della Scuola Storica ${ }^{5}$, nonostante il brillante valore letterario, non è superiore, da un punto di vista scientifico, ad un saggio disarticolato di un dilettante dell'Enciclopedismo.

Savigny è realmente il primo grande autore ad entrare a fondo nello studio delle fonti del Diritto. E per questo che i suoi insegnamenti devono meritare la nostra speciale attenzione, e, per una questione di facilità didattica, tenteremo di riassumere in paragrafi i punti maestri delle llezioni che egli imparte sulla materia ${ }^{6}$ Sarebbero i seguenti:

a) L'autore distingue le fonti delle relazioni giuridiche particolari (contratti) dalle fonti della regola giuridica generale (legge)

b) Fa una distinzione tra fonti di Diritto propriamente dette, e fonti storiche ${ }^{7}$

c) Sono fonti del Diritto il Popolo e lo Stato, ai quali corrispondono, rispettivamente, il Diritto Consuetudinario e la Legge.

d) Considera ancora, come fonte, il Diritto Scientifico, o Diritto dei Giureconsulti ("Juristenrecht"), che suddivide

3. HEINECIUS. Recitationes in Elementa Iuris Civilis secundum ordinem Institutionum, § $\$ 44$ e segg., Coimbra, 1817.

4. MeLlo FreIRe. Institutiones Iuris Civilis Lusitani, $\mathrm{I}, \& \mathrm{~V}$ e sp., Coimbra, 1853.

5. Montesquiev. Do espirito das leis. Edizioni di Cultura, 1945, v. PICARD, Edmond. O direito puro. Ed. portoghese, s.d.. p. 183.

6. V Sistema, p. 40 e segg.

7. Vedi, nello stesso senso, alexandre Correia e Gatetano Sciascia, Manual de direito romano, p. 12. 
in teorico e pratico. Teorico, è quello risultante dalla ricerca scientifica, pura e semplice. Pratico, quello suscitato da un problema giuridico concreto, inclusa, pertanto, la Giurisprudenza, nel senso più stretto della parola ${ }^{8}$

e) Un aspetto importante della dottrina delle fonti di SAVignY è il fatto che egli attribuisca una certa procedenza della consuetudine rispetto alla legge, il che può essere spiegato a causa del suo storicismo. Bisogna far risaltare anche la circostanza che egli consideri i giuristi come una élite che rappresenta il Popolo nell'elaborazione del Diritto. Tutto il Diritto proviene dalla Coscienza Popolare, ma i giuristi, che fanno parte del popolo stesso, vengono ad essere gli organi abilitati per elaborare il Diritto in nome del Popolo.

Evidentemente, il suo discepolo Puchta seguì le sue orme, como si può vedere del Corso delle Istituizioni.

Da allora in avanti, in modo generale, la dottrina delle fonti è stata esposta dai giureconsulti dei diversi paesi occidentali seguendo le teorie di SAvignY.

Tuttavia vi sono stati anche autori che, per fortuna, non si sono circoscritti a codesti limiti e, in un grande sforzo di rinnovamento, hanno apportato luci più chiare allo studio del tema di cui stiamo trattando. E quanto tenteremo di analizzare in seguito.

2 Studi Contemporanei particolarmente importanti, realizzati sulla Materia.

Bisogna innanzi tutto mettere in risalto la grande importanza di alcune monografie relative alla materia. Tra di esse possiamo citare quella di Henri de PAge, in Francia, A Propos du Gouvernement des Juges ${ }^{9}$, quella di Des Vecchio, in

8. Nella accezione di insieme dei pronunciamenti degli organi della funzione gludicante.

9. PAGE, Henry de. A propos du gouvernement des fuges. Parigi, 1931. 
ltalia, I Principi Generali del Diritto ${ }^{10}$, e, in Spagna, il recente studio di Puig Brutau, La Jurisprudencia como Fuente del Derecho ${ }^{11}$

Un significato tutto speciale, per l'approfondimento dell' argomento, ha per noi il Recueil d'Etudes sur les Sources du Droit en l'Honneur de François Gény ${ }^{12}$, al quale ha preso parte una pleiade di specialisti nella materia. L'opera, in tre volumi, racchiude saggi eccellenti su tre temi fondamentali: J. Aspetti Storici e Filosofici; II. Fonti Generali dei Sistemi Giuridici Attuali; e III. Fonti dei Diversi Rami del Diritto.

3. Opera di Gény. La maggior opera scritta fino ad oggi sulla materia è stata quella di colui in onore del quale furono pubblicati codesti studi, l'eminente Françors GìnY, il grande restauratore, nel Diritto Privato, della concezione classica del Diritto Naturale. Si intitola Méthode d'Interprétation et Sources en Drdit Privé Positif, 2 volumi ${ }^{13}$, e le sue conclusioni furono posteriormente completate da un'altra opera magistrale, la "Science et Téchnique en Droit Privé Positif", in quattro volumi ${ }^{14}$.

Profondamente significativo è il suo contributo nel settore della metodologia dell'interpretazione delle Fonti, e in quello della construzione scientifica del Diritto. Ma quanto alla questione dell'identificazione delle fonti e alle loro classificazioni, GENY rimane nel terreno tradizionale, distinguendo le fonti sostanziali dalle fonti formali, e, tra di queste, la

10. DeL Vecchio, Giorgio. Los principios generales ael derecho, trad. di J. Osorio Morales, Barcelona, 1933.

11. Puig Brutad, José. La jurisprudência como fuente del derecho. Barcelona, s.d.

12. Recueil d'tudues sur les Sources du Droit en l'Honneur de François Gény. Parigi, Sirey, s.d., 3v.

13. GENX, François. Méthode d'interpretation et sources du droit privé positif. 2.a ed. Parigi, 1919, 2 t. 4v.

14. Idem, Science et téchnique en droit privé positif, Parigi, 1922-1924, 
legge, il costume, la tradizione e l'autorità, comprendendo anche la dottrina e la giurisprudenza ${ }^{15}$

Inoltre, nella nota 1 , della pagina 239 , del tomo I del Méthode, lo stesso autore confessa di non poter far altro che rimettere il lettore agli autori dell'epoca, vale a dire GIERKE, Korkounov, Charmont, Sternberg e Kohler. 16

4. II Contributo dei Pubblicisti. Invece una vera rivoluzione si verificò, nella materia, grazie alle moderne teorie del Diritto Pubblico, professate da Duguit, Jèze, Bonnard e altri, specialmente per la premessa da essi stessi stabilita, e cioè che non esiste una separazione assoluta tra il Diritto Pubblico e il Diritto Privato, e che può esservi nulla di meno esatto dell'affermazione corrente, che "l'esprit qui doit présider à l'étude du droit public n'est pas le même que celui qui doit inspirer l'étude du droit privè" ${ }^{17}$.

La necessità di comprovare questa affermazione basica ha condotto codesti autori a fare una serie di revisioni dei concetti tradizionali, tra i quali, nella materia specifica, risalta, per importanza, tutto ciò che concerne gli atti giuridici ${ }^{18}$

Effettivamente, sebbene Bonnard affermi la differenza di contenuto tra il Diritto Pubblico e il Diritto Privato, deve riconoscere che "en ce qui concerne l'activité de l'État de ses organes, on pourrait à la rigueur concevoir que les règles du droit privé leur soient appliquées. Les actes juridiques de l' État seraient accomplis suivant les mêmes règles que ceux des particuliers" 19

Così è stato possibile arrivare ad una concezione monista

\footnotetext{
15. V.Méthode, I, p. 237 e segg.

16. Méthode, I, p. 239, nota 1.

17. Duguir, Léon. Manuel de droit constitutionnel. Parigi, 1907, p. 66.

18. Atto giuridico, secondo l'art. 81 del Codice Civile, è "ogni atto lecito che abbia come fine immediato quello di acquisire difendere, trasferire, modificare o estinguere diritti".

19. Bonnard, Roger. Précis de droit public. Parigi, 1939, p. 1. Cf. Duguit, op. cit., pp. 67-69.
} 
dell'atto giuridico, passando a considerarsi tali la legge, l'atto regolamentare, ecc. Ora, ciò stabilito, e posto che atti come la legge e il giurisdizionale costituiscano atti giuridici in relazione alle fonti del Diritto, non si poté fare a meno di rivedere le idee tradizionali, poichè, a rigore, la vera fonte del Diritto passerebbe ad essere lo stesso atto giuridico, nel cui concetto sarebbero racchiuse le più importanti fonti secondo la dottrina corrente.

5. Brethe de la Gressaye e Laborde-Lacoste. La disamina concreta e sistematica di queste nozioni, in materia di fonti del Diritto fu effettuata in modo eccellente da BRETHE de la Gressaye e Laborde-Lacoste, nella loro opera Introduction Générale à l'Étude du Droit. In essa sono state distinte due specie fondamentali di quella categoria giuridica: fonti atti e altre fonti. Fonti atti, la legge, il diritto corporativo, l'atto giuridico individuale e l'atto giurisdizionale. $\mathrm{E}$ altre fon$\mathrm{ti}$, la consuetudine, i principi generali di diritto e la dottrina ${ }^{20}$.

Il concetto di legge non ha bisogno di maggiori spiegazioni. Sarebbe l'atto giuridico legislativo. Diritto Corporativo, l'insieme speciale di norme che reggono le istituzioni sociali. Atto giuridico individuale, i contratti, i testamenti, ecc. Atto giurisdizionale, la giurisprudenza ${ }^{20 a}$.

Anche le altre fonti, a questo punto, possono prescindere da schiarimenti più minuziosi, giacchè torneremo ad esse durante questo nostro lavoro.

$\mathrm{Ma}$ per una questione di giustizia bisogna segnalare il fatto che l'idea degli atti giuridici come fonte di Diritto fu

20. LA GRESSAYe, Brethe de e LABORDE-LACOste. Introduction générale à l'étude du droit, p. 179, v. pp. 169-196.

20a. importante notare, in questo dettaglio, la saggezza dei Romani, che racchiudevano in un unico concetto, quello di "lex", queste tre categorie Esisteva cosi la "lex privata" (la clausola contrattuale, p. es., lex venditionii); la "lex collegii" (quella dele associazioni) e la "lex publica" secondo la nozione moderna. V. A. Correia e G. Sciascia, Manual, p. 14). 
divulgata, tra noi, da OrLando Gomes, nella sua Introduzione al Diritto Civile la cui esposizione è profondamente collegata con i capitoli di Brethe DE LA GRESSAYE e LABORDE-LACOSTE sulla stessa materia ${ }^{21}$

\section{Sostituzione della nozione di "fonte" con quella di "forma" del Diritto Positivo.}

v Improprietà della espressione "fonte" per designare i modi di espressione del Diritto. L'esame dei diversi autori che, da Savigny ai nostri giorni, hanno trattato il problema della specificazione e classificazione delle "fonti" del Diritto, mostra un certo progresso, non sempre intenzionale, riguardo alla graduale sostituzione della nozione di "fonte" con la nozione di "forma" del Diritto Positivo.

In verità, dato che la parola "fonte" serve a designare il fulcro generatore di qualche cosa, il suo uso in questo capitolo del Diritto ha dato luogo ad una serie di confusioni, poichè l'oggetto sono piuttosto i modi, le forme di espressione del Diritto e non le sue fonti di produzione, come si vede espresso chiaramente in alcuni autori.

Effettivamente la legge, il costume ecc., non generano, non creano, non producono il Diritto. Il Dirito é generato dalle necessità sociali e dalla volontà umana. E questa che, prendendo conoscenza delle imposizioni impostergabili della realtà sociale-giuridica, si serve della organizzazione politica della nazione, lo Stato, per creare la legge. Nello stesso modo, già sul terreno dei fatti (in contrapposizione a quello del diritto costituito) è ancora la volontà umana, conglomerata nella Coscienza Popolare ${ }^{22}$ che crea il costume.

21. Gomes, Orlando. Introdução ao direito civil, p. 59-62; cf. BRETHE DE la Gressaye e laborde-Lacoste, op. e loc. cits.

22. Usiamo codesta espressione per esprimere l'unità morale delle volontà individuali, e non l'idea di un essere autonomo, secondo l'insegnamento della Scuola Storica (v. Alexandre Correia, Concepção Histórica do Direito, p. 114-128) 
Così, in realtà le fonti del Diritto propriamente dette sono l'arbitrio umano e il Diritto Naturale. Lo Stato e la Coscienza Popolare (o il Popolo) sono solamente le cause strumentali della elaborazione del Diritto. Invece la legge, il costume, ecc. sono i modi, le forme, i mezzi tecnici dei quali si serve la volontà umana per esternare, far conoscere, oggettivare, attraverso lo Stato e la Coscienza Popolare, il diritto suscitato dalle imposizioni naturali della vita in società.

2. L'idea di "Fonte Formale" Questa nozione la troviamo abbozzata in molti autori, tra i quali ricordiamo FRANçOIs GÉNy, TRABucchi, Bonnecase ${ }^{23}$, Brethe de la GresSAYE e LABORDE-LACOSTE. Tra i nostri, possiamo nominare Paulino Neto ${ }^{24}$, Serpa Lopes ${ }^{25}$, Orlando Gomes ${ }^{26}$ e VICENTE RÁO ${ }^{27}$

Effettivamente, codesti giurisperiti adottano generalmente la divisione proposta da Fraçors GÉNY, che, come abbiamo visto, distingue le fonti sostanziali dalle fonti formali del Diritto. Fonti sostanziali sarebbero gli elementi, i dati del Diritto - materiali storici, razionali e ideali - ai quali le fonti formali - legge, costume, tradizione e autorità - danno una espressione appropriata. Ora, come si vede, i cosiddetti elementi sostanziali possono essere perfettamente ridotti al Diritto Naturale; le cosiddette "fonti formali", invece, non sono altro che un mero modo di esteriorizzazione dei precetti della giustizia applicati alla soluzione dei problemi sociali.

Per questo, l'espressione "fonte formale" é, secondo noi, doppiamente impropria, perché o noi vediamo la legge, il costume, ecc., dal punto di vista del loro contenuto, vale a

23. BonNeCASE, Jullen, v. Supplément, al Trattato teorico-pratico di Baudry-Lacantinerie, t.I, p. 396 e sp., Parigi, 1924; v. tb. Introduction à l'étude du droit. Parigi, 1939, p. 19.

24. Paulino Neto. Cadernos de direito civil, I. Rio, 1934, p. 35.

25. Serpa Lopes. Curso, I. 2.a ed. Rio, 1957, p. 65.

26. Gomes, Orlando, op. cit., p. 39. 275 .

27. Ráo, Vicente. O direito e a vida dos direitos, I. São Paulo, 1952, p. 
dire consideriamo il diritto stesso, già costituito, e non la fonte, o li consideriamo secondo il mero prisma della fonte, $e$ in tal caso, sarebbero solamente il modo attraverso il quale il diritto diviene positivo, si esterna, si esteriorizza ${ }^{28}$.

3. Ahrens e Fernandes Elias. Già due autori del passato ebbero una idea nitida di quanto stiamo esponendo. Essi sono il classico Ahrens, autore del Cours de Droit Naturel, e Fernandes Elias, giureconsulto spagnuolo, tra le cui opere contiamo il Trattato del Diritto Civile Spagnuolo. Del primo sono le seguenti parole, che troviamo conveniente trascrivere secondo l'originale: "Tout droit se manifeste donc dans la vie réelle dans des formes, soit dans des formes générales de naissance, comme les coutumes et les lois, soit dans des formes spéciales dans toutes les matières particulières" ${ }^{29}$. Sullo stesso diapason il secondo ci insegna: "Téngase muy en cuenta que nosostros aceptamos como fuentes del Derecho y de la ley a la legislacion, la política y la jurisprudencia, sola y exclusivamente en el terreno material, pratico y formulario, porque el Derecho y la ley no tienen, no pueden tener otras fuentes que la razón, la voluntad, la libertad y el bien y la justicia" ${ }^{30}$.

4. Necessità della distinzione tra "fonte" e "forma" del Diritto. Codesta distinzione non è nè oziosa, nè superflua. Per tre ragioni, che andremo esaminando a mano a mano che le indicheremo.

Innanzi tutto, essa corrisponde alla realtà dei fatti. Come abbiamo visto, la legge, il costume, ecc., in realtà non generano il diritto. Sono appena modi di espressione del Diritto.

28. D'altro canto, l'espressione è ibrida, poichè "fonte" è causa efficiente, e "formale", come il nome stesso dice, è causa formale, da non confondere con la prima.

29. Ahrens, Henri. Cours de droit naturel, I. Leipzig, 1875, p. 174.

30. Elias, Fernandes. Curso, I. Madrid, 1880, p. 58. 
In secondo luogo, essa serva ad evitare una serie di errori e di imprecisioni nel caso si vogliano specificare codeste categorie giuridiche.

In verità ci sembra che la confusione esistente tra le nozioni "fonte" e "forma" del Diritto sia la causa principale di diversi errori. Per esempio, quello di considerare l'equità come termine adottato normalmente dai diversi autori "fonte formale" Come si sa, l'Equità, in sé, è un principio simile a quello della giustizia, e, pertanto, può essere solo fonte generatrice e mai formale; dal punto di vista della Etica è una virtù, e, evidentemente nessuna virtù potrà essere considerata, a rigore; modo di espressione del Diritto. Sarà considerata solamente come un'abitudine pratica capace di procurare effettivamente ciò che è buono. Quindi è ben lontana dall'aderire alla nozione di forma di espressione del Diritto.

Un altro esempio di equivoco "data venia", ci è fornits da alcuni maestri quando considerano lo Stato "fonte formale" del Diritto. Nonostante le distinzioni che si cercano di fare, il fatto è che lo Stato non è altro che lo strumento che manda ad effetto la norma positiva ${ }^{31}$

La definizione rigorosa di concetti che si riferiscono alla forma del Diritto positivo sembra capace di evitare tutte codeste mancanze, dato che dilucida bene l'idea che, in detta materia, lo studioso deve attenersi solamente ai modi esterni di positività del Diritto.

In terzo luogo, invece di continuare a preoccuparci di escludere alcune forme e di accettarne altre, con una visione eccessivamente ristretta della questione, abbiamo notato che, grazie a codesto espediente dell'analisi giuridica, il campo che ci si apre è molto più vasto, giacchè, come si vedrà, esistono numerosi atti e fatti umani che solo possono essere considerati forme di espressione del Diritto, ma possono essere coordinati secondo i principi di una stessa sistematica.

31. RÁo, Vicente, op. cit., p. 277. 
In verità, oltre alla legge, al costume, alla giurisprudenza, alla dottrina, come abitualmente enumerano gli autori, sono considerati modi, attraverso i quali il diritto si rivela per integrare l'insieme delle relazioni sociali-giuridiche, il Diritto Estraneo, i Principi Generali del Diritto, il Diritto Statutario, gli Atti Giuridici Particolari, lo "Standard", ecc.

Come l'albero che, grazie alla potatura razionale, viene liberato dei rami superflui, questo capitolo della Scienza Giuridica, invece di diminuire, cresce in armonia e sostanza.

In seguito tenteremo di dimostrare come, una volta fissate queste nozioni, diventa possibile elaborare una classificazione generale delle fonti e delle forme del Diritto Positivo, in modo più chiaro e più completo.

\section{Classificazione delle forme di espressione del Diritto.}

I. Introduzione. La definizione del concetto di forma di espressione del Diritto, diversa da quella che riguarda le sue fonti propriamente dette (sia detto subito), non stabilisce un sezionamento di codeste categorie giuridiche tale da farle considerare su piani completamente separati. Sebbene, secondo noi (come pensiamo aver esposto prima), l'analisi giuridica debba fare una precisa distinzione di esse per una migliore conoscenza dell'effettivo contenuto delle rispettive nozioni, è giocoforza riconoscerne la interdipendenza, e ammettere che la delucidazione di una non può prescindere dallo schiarimento delle altre.

II. Il capitolo delle fonti del Diritto propriamente dette concerne quella che potremmo chiamare etiologia giuridica; il capitolo invece che si ocupa delle forme di espressione dei modi per i quali il diritto, latente nella natura delle cose, diventa, per un atto della volontà umana, oggettivamente definito e coercitivo ${ }^{32}$, è quello della morfologia del Diritto.

32. Cf. Alexandre Correia e Gaetano Sciascia, Manual de Direito Romano, I, p. 12 . 
Ma si vede chiaramente che la forma, per se stessa, non riveste una grande importanza se non è accompagnata dalla materia, dal contenuto che oggettiva e definisce. Ed è evidente che codesto contenuto, per arrivare ad acquistare una forma, è dovuto passare necessariamente attraverso un ampio processo di elaborazione, il cui studio si riferisce esattamente al capitolo delle fonti del diritto propriamente dette.

Ecco perchè, malgrado la distinzione, per noi indispensabile, tra fonte e forma del Diritto Positivo, tenteremo, riprendendo alcune nozioni già abbozzate precedentemente, di precisare bene la distinzione tra le diverse fonti del Diritto, per classificarne, dopo, le forme di espressione, impropriamente chiamate fonti formali.

Così, sarebbero quatro i tipi di fonti del Diritto, e cioè: le fonti storiche, le fonti genetiche, le fonti strumentali e le cosiddette fonti formali, per noi semplicemente forme di espressioni del Diritto.

2. Fonti Storiche. L'espressione fonte storica può essere interpretata in più di un senso. Uno di essi lo troviamo in SAvignY: l'accezione dell'insieme dei documenti che servono come base per l'elaborazione della scienza giuridica ${ }^{32}{ }_{-a}$ Un altro senso simile a questo, ma più comunemente utilizzato, é quello proposto da Correia e Sctascia, secondo il quale "si intendono per fonti di diritto i documenti attraverso cui arriviamo a conoscere il diritto di un determinato popolo" ${ }^{33}$.

Da parte nostra, ci teniamo ad osservare che codesti sarebbero solamente modi esterni di concepire fonti storiche. Vi sarebbe anche un altro modo di analizzarle, (questo di natura interna); secondo esso si indicherebbe, con questa espressione, il processo storico della norma giuridica.

32a. Savigny, Sistema, I, p. 41.

33. Correia e Sciascia, op. cit., p. 12. 
Codesto processo storico, potrebbe essere suddiviso in prossimo e remoto. E prossimo, quando le condizioni di natura sociale-giuridica sono state tali da rendere possibile, in un dato momento, la elaborazione della legge o altra forma di espressione del diritto. E remoto, quando si riferisce alle origini storiche della istituzione giuridica che la norma si propone di regolamentare. Sotto questo prisma, lo studio delle fonti può risalire ai più remoti (e non sempre meno importanti) fattori che contribuirono alla formazione di un popolo o di una cultura.

Lo studio delle fonti storiche delle istituzioni giuridiche è indispensabile per il loro effettivo conoscimento. Come osserva Sternberg, "colui che volesse realizzare il Diritto senza la Storia non è nè giurista nè utopista; non darà vita a nessuno spirito di ordinamento sociale cosciente, ma a puro disordine e a pura distruzione" ${ }^{34}$.

Per quanto riguarda la sua correlazione con lo studio degli altri tipi di fonti, si deve notare che è la storia del Diritto quella che rende possibile una conoscenza migliore delle sue fonti, sia genetiche, sia strumentali.

3. Fonti Genetiche. Per fonti genetiche del Diritto intendiamo il Diritto Naturale ${ }^{35}$ e l'arbitrio umano.

Circa ciò che chiamiamo Diritto Naturale, bisogna dare alcuni schiarimenti.

Innanzi tutto, prendiamo l'espressione nel suo senso classico, nel senso aristotelico-tomista, e non in quello utiliz-

34. Sternberg, Theodor. Introducción a la ciencia del derecho, $2 .^{\mathrm{a}}$ ed. Labor, 1940, p. 32 .

35. Sul concetto di Diritto Naturale, v. specialmente la tesi del Prof. ALEXANDre CoRREIA: Esiste un diritto naturale? Quale il suo concetto? 1917; dello stesso autore, $\nabla$. ancora Concezione Tomista del diritto naturale. A Balança tomo II, n. ${ }^{\circ} 32$. V. CAthrein: Philosofia Moralis. Barcelona, 1945, nn. 295-297, ecc. V. 11 nostro studio Diritto naturale e diritto positivo. Estudos Juridicos in commemorazione del cinquantenario della Revista dos Tribunais. 
zato dai giusnaturalisti, da Grotius a Rousseau. Secondo questi autori, il Diritto Positivo non dovrebbe essere altro che la proiezione di un ordine prestabilito nella stessa natura delle cose, portata a termine, a rigore, indipendentemente dall'arbitrio umano. Il quale, promulgando una legge, sarebbe soltanto un puro strumento di codesto Diritto Naturale determinista, necessario e immutabile ${ }^{\mathbf{3 6}}$

Già secondo la concezione classica del Diritto Naturale, questo diritto, sebbene fondato su principi stabili, non può non soffrire un mutamento costante, in dipendenza dalle condizioni dell'ambiente e del momento. Così, mentre il Giusnaturalismo ha creato un Diritto Naturale sostanzialmente cerebrale, deduttibile "more geometrico", secondo la Scuola Classica, il diritto deve essere elaborato d'accordo con la realtà dei fatti ${ }^{37}$

Un'altra distinzione da fare è quella relativa al fatto di considerare il Diritto Naturale nella sua accezione lata. Secondo un concetto più stretto, il Diritto Naturale si riduce ai primi principi della giustizia; da un punto di vista più ampio, il Diritto Naturale comprende non solo codesti principi stabili, ma anche le necessità sociali che, presi in considerazione gli imperativi oriundi da codesti principi, emergono dallo stesso evolversi dell'esistenza e della cultura umana.

Effettivamente, i mutamenti del processo, le trasformazioni della scienza, la transmutazione accidentale delle men-

36. Grotus, Hugo. De Iure Belli ac Pacis, Prolegom. 6-11; Cap. I, X, 1-7; I, XIV; I, I, 1-5 ecc., Haia, Ed. de Telders, 1948; Roussead. Contrato Social, LI. Ed. Cultura, 1944.

In Economia Politica, il giusnaturalismo si manifesta nella Fisiocrazia, di QUESNay e TuRgot. Il primo é autore di un trattato di Diritto Naturale, (v. Papaterra Limongi. Economia Política. 5.a ed. 1959, p. 119).

37. V. le opere citate di Alexandre Correia e Catherein. V. anche San Tommaso d'AQUINo. Suma Teologica, 1.a, IIas. Q. XCV. Arts. III e IV; dello stesso autore, v. in: Aristotelis Starigitae Libros Nonnullos Commentaria, V. lec. 12, in: Thomae Aquinatis Opera Omnia. Parigi, v. XXV, p. 460, 1875; MARITAIN, Jacques. Humanismo integral, p. 16 e segg.; SouzA, José Pedro Galvão de. O positivismo jurídico e o direito natural, San Paolo, 1940. 
talità, vanno creando una serie di necessità che bisogna considerare senza pregiudicare gli imperativi della giustizia.

Alcune istituzioni esigono trasformazioni radicali, altre spariscono perchè cadute in disuso, altre ancora vanno sorgendo ed è necessario regolamentarle. Ecco, pertanto, le necessità sociali, le necessità che, in virtù della natura stessa dell'uomo e delle cose, devono essere soppresse - a generare la regola del diritto positivo.

D'altro canto, codesta regola non si esprime da sè, nè può essere identica in luoghi e momenti differenti. Bisogna, quin$\mathrm{di}$, valutare le convenienze della sua applicazione in questo o in quel modo, o anche considerare se non sarebbe meglio differirne la promulgazione ad un'occasione più opportuna. Considerazioni di questa natura, insomma, concernono l'arbitrio umano nella genesi del Diritto Positivo, sebbene codesto stesso arbitrio debba essere subordinato sia ai principi primi di giustizia, sia alla loro diversa condizione.

4 Fonti Strumentali. Ora, la volontà umana, quantunque conservi sempre il carattere essenziale di volontà individuale, ha bisogno, per realizzare la coercitività della legge e di altro precetto giuridico, di agire attraverso gli organi che personalizzano l'organizzazione sociale di un popolo singolo, o dei popoli nel loro insieme universale. Codesti organi, secondo noi, sono di due nature. Una giuridica, nella sua accezione stretta, un'altra, di fatto, sostenuta appena dalle imposizioni della propria realtà sociale.

Sul piano giuridico, l'organo che rapresenta l'unità è lo Stato; sul piano dei fatti, la Coscienza Nazionale. Proiettati nel campo delle relazioni tra i popoli, al primo corrisponde la Società Politica delle Nazioni, e al secondo la Coscienza Socrale Universale.

Tali entità sarebbero a rigore le vere fonti strumentali del Diritto Positivo, alle quali corrisponderebbero, in modo 
precipuo e generico, la legge e il costume, incluso gli usi internazionali.

Oltre ciò, potremmo allineare, al lato di queste, una terza categoria, vale a dire, l'accordo delle parti, che è, realmente, il mezzo a cui si afferrano gli interessati per creare obblighi e diritti, sul piano delle questioni particolari ${ }^{38}$

Esposta la nozione di queste fonti del diritto, secondo la accezione propria del termine, possiamo passare adesso, alle fonti impropriamente dette formali, o forme di espressione del Diritto Positivo, oggetto precipuo di questo lavoro.

5 Fonti Formali (impropriamente dette) o Forme di Espressione Del Diritto. Classificazione secondo il criterio della natura della coercitività. Se è vero che le fonti storiche, nel lcro aspetto interno, costituiscono l'immenso crogiolo dalle cui complesse ebollizioni emergono le necessità sociali; se è assodato che sono codeste necessità, considerate e manipolate dalla volontà umana, a generare la norma giuridica; se è evidente che per oggettivare i vincoli del Diritto gli uomini si servono di entità capaci di rendere efficaci questi vincoli, ci appare chiaro anche che tutte codeste fasi del sordo e multiforme processo di elaborazione dell'insieme delle relazioni giuridiche positive troverà la conclusione definitiva nelle forme attraverso le quali il Diritto si rivela e acquista capacità coercitiva.

Le suddette forme, seguendo il criterio che potremmo chiamare della natura della coercitività, possono esse inizialmente classificate in tre categorie: $1 .^{\mathrm{a}}$ ) degli atti giuridi-

38. Si osservi che lo stesso Codice Napoleonico ha consacrato il principio generale che "les conventions légalement formées tiennent lieu de LOI à ceux qui les ont faites" (art. 1.134). D'altro lato abbiamo visto la tendenza moderna nel considerare tanto gli atti giuridici in generale, quanto le "fonti" - del Diritto. E per finire abbiamo notato (nota 20-a) come 1 romani denominavano "leges privatae" le clausole contrattuali. 
ci; $\left.2 .^{a}\right)$ degli atti sociali di fatto, con forza giuridica; $\left.3 .{ }^{a}\right)$ delle conclusioni della scienza giuridica.

A -Atti Giuridici. Con l'espressione atti giuridici noi vogliamo intendere non solamente le questioni particolari ${ }^{39}$, ma l'atto giuridico nella sua più ampia accezione, secondo quanto ci hanno insegnato i pubblicisti ${ }^{40}$

Così vi sarebbero inclusi: a) la legge; b) l'atto giurisdizionale considerato in particolare; c) il diritto corporativo o statutario; d) il diritto estraneo; e) l'atto giuridico amministrativo; e f) gli atti giuridici individuali.

Il termine legge è a sua volta inteso in senso lato ${ }^{41}$, inmodo da comprendere anche i decreti, i regolamenti e gli atti amministrativi, come le sentenze di I e II grado, circolari, risoluzioni, ordini di servizio, ecc., che venissero a risolvere ingiunzioni di carattere generale, seppure nell'ambito di un settore particolare ${ }^{42}$

Atti Giurisdizionali sono quelli resi effettivi dal Potere Giudiziario, di fronte ad un problema giuridico, dal carattere contenzioso. In verità le sentenze, in virtù delle proprietá della "res judicata", che "pro veritate accipitur" 43 , sono una delle forme di cui si riveste il diritto, una volta che tali atti, sebbene subordinati al diritto pre-esistente, siano capaci di definire situazioni giuridiche anteriormente dubbie e che, a partire dagli stessi, passino a regolare una parte del complesso generale delle relazioni sociali-giuridiche.

39. SERPA LOPES. Curso de direito civil, I. 2.a ed. 1957, p. 39 e segg.

40. V DUGUIT. Cours de droit constitutionnel, p. 64 e segg.; Bonnard. Précis, p. 1; v. ancora, de DUGणIT: Les transformations au droit public. Parig1, 1921, pp. 75-146.

41. V. ChIRonI, Istituzioni, I, p. 19.

42. Per esempio, il regolamento interno del settore di benefici della autarchia della previdenza sociale. Include norme di carattere generale, dentro di un settore particolare.

43. Digesto, Libro XVII, 207, frag. de Ulplano. 
Per diritto corporativo o statutario comprendiamo l'insieme delle relazioni giuridiche che reggono o le persone morali o i corpi sociali che fanno da intermediari tra l'individuo e lo Stato. Esso presenta una natura speciale, perchè, secondo la terminologia di $\mathrm{C}_{A T H R E I N}{ }^{44}$, codeste società sono imperfette, incapaci di bastare a loro stesse.

Non vi sono incluse le cosiddette (impropriamente) autarchie parastatali, o dipartimenti del Diritto Francese, perchè li consideriamo organi del proprio Stato, puro frutto del decentramento amministrativo, allo stesso modo in cui le province costituiscono il risultato del decentramento politico-territoriale.

Questo Diritto comprende non solamente le società di Diritto Privato, civili e commerciali, ma anche quelle di Diritto "Sociale", quali i sindacati e le associazioni professionali. A questi sono strettamente legati anche atti giuridici, per es. i contratti collettivi di lavoro, che secondo DugurT, appartengono alla specie delle considdette leggi-convenzioni ${ }^{45}$

Sono ancora atti giuridici quelli concernenti il cosiddetto Diritto Estraneo. Suddividiamo questo Diritto in: Diritto Romano, Diritto Canonico, e Diritto delle Nazioni Moderne, espressione questa, utilizzata dalla Legge della Buona Ragio$n \mathrm{e}^{46}$ e adottata dai civilisti del passato ${ }^{47}$

Sebbene, come la stessa espressione indica, si tratti di un diritto diverso dal nostro, prodotto da poteri che non si confondono con quello dello Stato nazionale, esso abbraccia leggi che, ancora oggi, seppure in misura minore, integrano il nostro sistema di diritto positivo. E se ciò avviene è principalmente perchè sono stati creati come diritti operanti nelle

44. Cathrein, Philosophia Moralis, § 511, p. 355.

45. Duguit, Les Transformations au Droit Public, p. 129.

46. Del 17 agosto 1769 , ispirata da Pombal.

47. V p. es. COElfio da ROCHA, I, p. 22. 
rispettive società a cui corrispondono o a cui hanno corrisposto.

Quanto all'atto giuridico amministrativo bisogna considerare che differisce di molto dai regolamenti e dalle altre norme di carattere generale di iguale natura. In verità, con questa espressione vogliamo indicare, in modo specifico, gli atti amministrativi particolareggiati, come per esempio le risoluzioni registrate nei quali interviene lo Stato, ecc. Nello. stesso concetto includiamo gli atti di giurisdizione volontaria, in cui non esiste azione propriamente detta, ma pura richiesta, non esistono parti (autore e reo), ma solo interessati non esiste lite, ma questione ${ }^{48}$.

Per finire, dentro lo stesso ampio concetto di atto giuridico come forma di espressione del Diritto, viene compreso l'insieme delle questioni giuridiche particolari, che, evidentemente, non costituiscono norme di carattere generale; ma, dettando legge tra le parti, racchiudono, nella loro enorme massa, una trama di complessissime relazioni giuridiche sulla cui base riposa tutto l'insieme delle situazioni che la legge suppone come ipotesi generale, ma alle quali solo codesti atti possono imprestare vitalità concreta e operante.

Pertanto sono anche esse forme attraverso cui il diritto si rivela. E sebbene particolari, sebbene ristrette alle parti contraenti, esse sono nel loro immenso acervo, e senza alcun dubbio, tra i modi più importanti di cui ci serviamo perchè il Diritto sia oggettivato e assuma forza coercitiva.

Passiamo adesso a quella categoria che proponiamo venga denominata "atti sociali di fatto con forza giuridica", o, più semplicemente, "atti con forza giuridica".

48. MARQUES, José Frederico. Ensaio sobre a jurisdição voluntária, 1952, p. 200; REzENDE FILHo, Gabriel de. Curso de direito processual civil, III. São Paulo, 1946, p. 58, § 853.; v. anche codesta monografia Do nome civil das pessoas naturais, pp. 392 e 417 , note 644 e 684 . 
B - Atti Sociali di Fatto, con Forza Giuridica. Cercheremo di spiegare, preliminarmente, il perchè di codesta denominazione e il perchè dell'aggruppamento di tali atti in una categoria a parte.

Esaminando la classificazione delle "fonti formali", proposta da Brethe de La Gressáye e da Laborde-Lacoste, quantunque ne avessimo verificato la procedenza generica, abbiamo notato che, in verità, gli autori, non si limitano, ad un criterio unico, o meglio, non completano la loro classificazione secondo i dettami del criterio adottato.

Realmente, come è già stato visto, essi dividono le fonti in fonti-atti e altre fonti, includendovi il costume, i principi generali del Diritto e la dottrina.

Ora, da parte nostra, con la dovuta venia, segnaliamo che, se si prende come punto di partenza l'atto giuridico, (il quale realmente procede, perchè il diritto è effettivamente frutto di un atto generato dalla volontà umana), bisogna vedere in codeste "altre fonti" ciò che esse presentano in comune con l'atto giuridico, e ciò che esse presentano di dissimile, per poter giungere, in questo modo, a una classificazione la più completa possibile.

Da qui la nostra proposta di distinguere, tra le "altre fonti" un gruppo specifico: quello degli atti sociali di fatto con forza giuridica; cioè il diritto del costume, in modo generále, o il costume giudiziario, diverso dall'atto giurisdizionale puro e semplice) e lo "standard" giuridico.

Atti sociali, perchè valgono in quanto generati dalla coscienza nazionale, per la soluzione di problemi propri alla vita dell'uomo in società.

Atti sociali di fatto, per contrapporli agli atti giuridici, poichè, mentre questi sanciscono l'ordinamento, quelli esercitano la forza coercitiva per una pura questione di "opinio necessitatis" 
Atti sociali di fatto con forza giuridica, poichè, sebbene non siano oggetto di norma giuridica specifica, sono capaci di operare come vera regola legale.

In codesto gruppo di atti, come è stato visto, abbiamo incluso subito, per eccellenza, il diritto consuetudinario, creazione tipica della volontà popolare, per la soluzione immediata, anteriore o complementare a quella della legge, dei problemi sociali-giuridici che questa regola non prevede e che tuttavia devono fondarsi su una regola generale.

Segue il costume giudiziario o giurisprudenza, diverso dall'atto giurisprudenziale considerato in particolare. Infatti, mentre questo ultimo costituisce un atto giuridico nella precisa accezione del termine, il costume giudiziario passa ad aveI $\in$ efficacia coattiva per una pura questione di opinione collettiva di necessità tra $\mathrm{i}$ giudicanti. In paesi simili al nostro non esiste legge che protegga il vincolativo generale delle decisioni della giurisprudenza; pertanto, se un tipo di decisione si ripete con religiosa costanza, un anno dopo l'altro e nella maggioranza dei tribunali, è giocoforza ammettere che difficilmente un magistrato $o$ un collegio giudicante cercherà di allontanarsi da questo orientamento, a meno che non intervengano fatti di forza maggiore.

, Consideriamo della stessa natura lo "Standard" giuridico, categoria importata dalla "Common Law" e che consiste in un criterio basico di valutazione di certi concetti giuridici indefiniti, variabili nel tempo e nello spazio ${ }^{49}$. Lo "Standard", o. direttiva giuridica, può essere legale, come quello del $\$ 6 .^{\circ}$ dell'art. 15 della Legge dell'Inquilinato, che include, nell'espressione uso proprio la obbligatorietà, per il proprietario, di rimanere per lo meno un anno nell'immobile liberato; può

49. P. es. il concetto della buona fede, di "bonus pater famillas", di pubblica, necessità normali del laboratore, ecc. (v. arts. 141 e 157 della Costituzione Federale Brasiliana, ecc.) 
anche dipendere dalla giurisprdenza, seguendo l'orientamento secondo il quale veniva considerato abuso di dirittol da parte del locatario, il pagamento della mora dinanzi al giudice per più di tre volte. $\mathrm{E}$ può essere semplicemente consuetudinario, come la generalità dei costumi di natura interpretativa ("secundum legum").

Lo "Standard" legale, è, insomma, legge per natura. Ma quello attinente alla giurisprudenza e quello semplicemente consuetudinario non sono che puri atti di fatto con forza giuridica.

Passiamo adesso all'ultimo tipo di forma di espressione del Diritto, secondo il criterio della natura della coercitività.

C - Conclusioni della Scienza Giuridica. Effetivamente, il Diritto su cui gli scienziati indagano nelle biblioteche non ragguaglia solamente sulla legge, il costume, la giurisprudeenza, ecc., ma possiede un suo proprio valore come forma di espressione del Diritto, latente nella natura delle cose.

Non senza delle forti ragioni, Savigny considerava la Scienza Giuridica una branca del Diritto Consuetudinario. Preferiamo considerarla come una scienza a parte, data la sua speciale elaborazione e la proeminente importanza come interprete principale del Giusto Diritto.

In verità, se da una parte le leggi ingiuste non sono propriamente leggi, ma puri atti discrezionari di poter $^{50}$, che non obbligano in coscienza, dall'altra, per raggiungere la legge giusta, è necessario ascoltare esattamente i reclami delle necessità sociali, sotto l'egida della giustizia, lavoro codesto che solo agli specialisti della Scienza del Diritto è dato realizzare con l'indispensabile sicurezza.

50. Sulla questione delle leggi ingiuste, v. Cathrein, op. cit., p. 182; Roubier. Théorie générale du droit. 2.a ed. 1951, p. 323.; RENNARD, Georges. Introduccion al estudio del derecho, v. I, p. 142; GÈNY, François. Science et téchnique, v. II, p. 348 . 
Nel Diritto Scientifico abbiamo incluso i Principi Generali del Diritto e i Dettati Giuridici, perchè è la Scienza quella che definisce tali principi; principi che, a loro volta, vengono espressi non raramente attraverso paremie o dettati di Il grado ${ }^{51}$.

Abbiamo dato il nome di natura della coercitività al criterio adottato per la classificazione che abbiamo appena tentato di fare. La ragione di ciò, come a questa altezza apare ormai chiaro, risiede nella circostanza che, mentre l'efficacia vincolativa degli atti giuridici proviene, in linea immediata, dalla sanzione statale, quella degli atti sociali di fatto, con forza giuridica, fluisce da altri fattori, come, per esempio, nel caso del costume, dalla "opinio necessitatis".

A sua volta, il Diritto Scientifico si impone, a volte, come un vero precetto, grazie al consenso dei dottori ("communis opinio doctorum") e alla sua corrispondenza con la verità giuridica.

Altri criteri, tuttavia, possono essere utilizzati per la classificazione delle forme di espressione del Diritto: per esempio l'importanza che presenta nella integrazione del sistema giuridico. Da questo punto di vista si distinguerebbe inizialmente la Legge, che è la forma fondamentale ${ }^{52}$, considerandosi forme complementari tutte le altre; tra di esse - il Costume, la Giurisprudenza, il Diritto Scientifico, i Principi Generali del Diritto e i Dettati Giuridici.

51. V. dello stesso R. Lmmongi França, Dos Principios Gerais de Direito, 260 p., 1963, e Brocardos Jurídicos - as Regras de Justiniano, 160 p., 1961, ed. della Revista dos Tribunais.

52. V. l'art. 4.' della Legge di introduzione al Codice Civile; v. anche l'art. 141, n. 2, della Costituzione Federale Brasiliana. 